\title{
FACTORES ASOCIADOS CON EL ESTADO NUTRICIONAL DE LOS NIÑOS Y NIÑAS EN EL PERÜ
}

\author{
Ysela Agüero P. ${ }^{1}$, Emma Cambillo $M .^{2}$
}

\begin{abstract}
Resumen: El objetivo del estudio fue identificar los factores asociados con el estado nutricional de los niños/niñas menores de 5 años en el Perú. Se consideró una muestra de 8103 niños/niñas menores de 5 años. Se ajustó un modelo MLG logit y se encontró que los factores significativamente asociados con la prevalencia de desnutrición crónica son el peso al nacer (OR:2,3; IC:1,8-2,8), la presencia de anemia severa (OR:2,3; IC:1,8-2,8), hogares pertenecientes a la zona rural (OR:1,1; IC:1,1$1,5)$, esta prevalencia se incrementa significativamente con el orden de nacimiento, la pobreza, juventud, bajo nivel de instrucción de la madre. La mayoría de los factores de riesgo identificados pueden ser controlados con la disminución de la pobreza, un adecuado control pre natal y una mejora de la calidad de vida y la educación de las madres, especialmente en la zona rural.

Palabras clave: Estado nutricional, peso, talla, edad, modelo logit. Abstract: The objective is identify associated factors with nutritional status of under 5 years children in Peru. Was considered a sample of 8103 children under 5 years. Fits GLM logit model, and found that factors significantly associated with the prevalence of chronic malnutrition are birth weight (OR:2,3; IC:1,8-2,8), severe anemia presence (OR:2,3; IC:1,2-4,5), in rural zone households (OR:1,1; IC:1,1-1,5), this prevalence significantly increases with birth order, and poverty, youth, and the low education of the mother. The majority of identified risk factors can be controlled with the reduction of poverty, adequate prenatal monitoring and improved quality of life and education of mothers, especially in rural areas.
\end{abstract}

Keywords: Nutritional status, weight, height, age, logit model.

\section{Introducción}

La nutrición en los primeros años de vida juega un papel importante en el crecimiento físico y el desarrollo intelectual del ser humano. Una adecuada nutrición va a favorecer tanto el crecimiento corporal del niño, como el desarrollo de sus capacidades cognoscitivas y lo hará más resistente a las enfermedades. Por el contrario, la desnutrición, especialmente en la infancia, constituye un impedimento para que los individuos y las sociedades desarrollen todo su potencial. Los niños y niñas desnutridos tienen una menor resistencia a las infecciones y más posibilidades de morir a causa de enfermedades comunes de la infancia, como las EDAs y las IRAs. Aquellos que consiguen sobrevivir quedarán atrapados en un círculo vicioso de deficiencias en su desarrollo físico y mental con un daño -en la mayoría de los casos, irreversible - y con enfermedades recurrentes, que le impedirán salir de la pobreza.

Según la UNICEF, la desnutrición es una emergencia silenciosa e invisible y por lo mismo muy grave; que cobra un precio muy alto en los niños y sus familias y que es resultado de un gran número de causas entre las que podemos destacar la carencia de alimentos debido a la pobreza, las infecciones comunes en los niños y niñas en los primeros años de vida, como las enfermedades diarreicas agudas (EDAs) y las infecciones respiratorias agudas (IRAs), pero, también tiene relación con los conductas o prácticas de salud de las madres, tales como su comportamiento

\footnotetext{
${ }^{1}$ UNMSM, Facultad de Ciencias Matemáticas

${ }^{2}$ UNMSM, Facultad de Ciencias Matemáticas
} 
con respecto a la lactancia exclusiva, la ablactancia, la higiene y el tratamiento de las EDAs y de las IRAs [10]. Estos comportamientos y prácticas a su vez son producto de un escaso acceso a la información y a los servicios de salud, a su bajo nivel de instrucción, y a que residen en lugares de baja densidad poblacional donde la atención que reciben de parte del Estado es muy deficiente.

De acuerdo con el Nuevo Patrón Internacional de Crecimiento Infantil, referido a lactantes y niños pequeños, presentado por la OMS en abril de 2006, la leche materna es esencial para el desarrollo, puesto que, todos los niños y niñas del mundo tienen el mismo potencial de crecimiento en talla, peso y coeficiente intelectual, por lo que, más que factores genéticos o étnicos, lo que determina el desarrollo sano de un niño hasta los cinco años es la nutrición, las prácticas de alimentación, el medio ambiente y la atención sanitaria. [11]

La buena nutrición tiene importantes repercusiones económicas en un país. Cuando una población está bien nutrida, la productividad individual es más alta, los costos de atención en salud son más bajos y el rendimiento laboral es mayor.

Si bien los ocho Objetivos de Desarrollo del Milenio de la ONU están orientados a mejorar las condiciones de vida de la población, el objetivo de reducir la pobreza a la mitad y reducir la mortalidad de los niños menores de cinco años, son los que están directamente relacionados con el tema de la nutrición. Los demás objetivos relacionados con la salud materna y la lucha contra enfermedades endémicas -también tienen mucho que ver con la nutrición, pues un organismo desnutrido es mucho más vulnerable a las enfermedades. La desnutrición afecta el rendimiento escolar y la asistencia de los niños y niñas a la escuela, Por lo tanto, es de vital importancia encontrar una solución al problema de la desnutrición, si se quiere cumplir con los objetivos sobre educación (ODM 2) y sobre la igualdad entre los géneros (ODM 3).

La mala nutrición energético-proteica-nutrimental se emplea para describir una variedad amplia de estados clínicos que oscilan desde los estados marginales y leves hasta los muy graves. En la actualidad, es el problema de salud más importante de los países en vías de desarrollo. Organismos internacionales como la OMS y la FAO la sitúan en cifras que sobrepasan los 800 millones de habitantes, de los cuales más de la mitad son niños [1].

El Perú es uno de los 189 países que se comprometieron a alcanzar los objetivos del Milenio, los cuales se centran en el ser humano y que, al mismo tiempo, deben ser logrados clentro de un plazo definido para lo cual son fácilmente mensurables. Uno de esos objetivos es reducir la desnutrición infantil.

En un documento publicado por el CIDE-INEI se da cuenta que, en el Perú, si bien la tasa de desnutrición crónica en niños, experimentó una pronunciada reducción desde 1984 a 2007, a partir de ese año, no ha sufrido cambios y se encuentra alrededor de $25 \%$ a nivel nacional a pesar de los esfuerzos por reducirla. El informe en cuestión hace énfasis en las prácticas de lactancia, de tratamiento de EDAs e IRAs y los hábitos de higiene, para explicar este estancamiento [2]. Pero, aparentemente existen otras causas que no están siendo abordadas y que podrían explicar este estancamiento y que nos llevan a tratar de buscar respuestas, tanto a partir de la revisión de las experiencias de otros países como del análisis de las bases de datos de encuestas realizadas en nuestro país y que se encuentran disponibles [3].

Se pueden mencionar muchas causas que en forma directa e indirecta contribuyen a la existencia de los problemas nutricionales. Entre estas se pueden indicar, como causas directas, el consumo de una dieta inadecuada y la presencia de infecciones que interfieren con la utilización adecuada de los nutrientes. Las causas indirectas están relacionadas estrechamente con el índice de pobreza e incluyen la insuficiente disponibilidad de alimentos en cantidad y calidad, inequidad en el acceso a los alimentos, conductas desfavorables de los miembros de la familia, en particular los prestadores de cuidados, las cuales redundan en prácticas inadecuadas de alimentación, falta de acceso y mala calidad de los servicios de salud, falta de información y educación [8].

A partir de la información obtenida mediante la revisión bibliográfica y tomando en cuenta que las tasas de desnutrición de niños y niñas menores de 5 años en el Perú, no están teniendo el descenso esperado a partir de los programas de salud ejecutados con miras a alcanzar los 
Objetivos de Desarrollos del Milenio, postulamos que los factores asociados con la desnutrición; no sólo están asociados con las prácticas de lactancia y el control de las enfermedades infantiles, sino que tienen que ver con las características del niño, de la madre y con su entorno.

Por todo lo mencionado, es importante contribuir mediante la investigación a una mejor comprensión de los factores de riesgo asociados con la desnutrición en los de los niños y niñas menores de 5 años, de modo que; se pueda alcanzar a las autoridades competentes información que los ayude a tomar las decisiones pertinentes en salud, economía y educación lo que contribuirá a que los niños y niñas peruanos lleguen a ser adultos saludables y productivos lo cual facilitará que salgan del círculo de la pobreza y que nuestro país tenga un verdadero desarrollo y no sólo en el discurso de nuestros gobernantes de turno.

El objetivo de la presente investigación es identificar los factores asociados con el estado nutricional de los niños y niñas menores de 5 años en el Perú. Esto es muy importante por que permitirá identificar aquellos factores que son de riesgo de desnutrición crónica y así contribuir al conocimiento de este problema. Por otro lado, la presente investigación también tiene un fin metodológico, pues, permite aplicar una técnica de análisis de datos estadísticos como son los modelos lineales generalizados (MLG) y dar uso a una base de datos que se encuentra disponible y que no está siendo suficientemente explotada. ${ }^{3}$

\section{Método}

El presente es un estudio de tipo descriptivo correlacional, de corte transversal. La población está conformada por los niños y niñas peruanos sobrevivientes de 5 o menos años que cumplan con los criterios de inclusión y exclusión establecidos en las ENDES (Programa Measure DHS+). La muestra está constituida por un total de 8103 niños con edades de cero a 59 meses y sus respectivas madres, constituyendo una sub muestra del total estudiado en la Encuesta Demográfica y de Salud familiar - ENDES 2009 [3].

Para evaluar el estado nutricional se emplearon las mediciones del peso (en gramos), talla (en centímetros) y edad (en meses). Para la expresión e interpretación de los datos antropométricos se utilizaron los índices $\mathrm{Z}$ del peso para la talla $(\mathrm{P} / \mathrm{T})$, talla para la edad $(\mathrm{T} / \mathrm{E})$, peso para la edad $(\mathrm{P} / \mathrm{E})$ e índice de masa corporal (IMC), calculados de acuerdo a las recomendaciones de la OMS [7].

Los factores asociados con el estado nutricional de los niños menores de 5 años se agruparon en tres dimensiones: Factores socioeconómicos, medioambientales y biológicos. Las asociaciones se evaluaron mediante indicadores bivariados y el ajuste de un modelo lineal generalizado logit.

Se identificaron las preguntas de la ENDES 2009 relacionadas con las variables de investigación, tales como, estado nutricional, factores sociales, económicos, ambientales y factores biológicos; previamente operativizadas.

Se realizó una revisión de las técnicas estadísticas relacionadas con el análisis multivariado de conjuntos de datos en los cuales se intenta determinar los factores que influyen en el estado nutricional de los niños y niñas, encontrandose que es posible utilizar algún miembro de la familia de modelos lineales generalizados (MLG) que describimos brevemente a continuación. Los modelos lineales generalizados (MLG) constituyen una familia ampliada formada por los modelos lineales clásicos y modelos en los cuales la variabilidad aleatoria puede tener cualquier distribución de probabilidades diferente de la Normal, siempre que, sea miembro de la familia de distribuciones de probabilidad Exponencial (binomial, Poisson, gamma, weibull, or inverse-Gaussian, etc.).e involucran transformaciones de la esperanza matemática de la variable respuesta (media poblacional) a través de funciones denominadas "Funciones Enlace" [6]. La generalización es posible por que todos los modelos incluidos tienen tres componentes:

\footnotetext{
${ }^{3}$ Las Encuestas Demográficas y de Salud Familiar - ENDES (Programa MEASURE DHS+), se realizan en los países en desarrollo, con el auspicio de la Agencia de los Estados Unidos para el Desarrollo Internacional (USAID). Uno de los objetivos de este programa de encuestas de Demografía y de Salud es proporcionar bases de datos y análisis a los organismos ejecutivos en el campo de la población para facilitar la consideración de alternativas y la toma de decisiones bien informadas.
} 
1. Una variable dependiente $(Y)$ es una variable aleatoria con distribución de probabilidades miembro de la familia Exponencial y cuya esperanza es dada por:

$$
E\left(Y / X_{1}=x_{1}, \ldots, X_{k}=x_{k}\right)=\pi .
$$

2. El predictor lineal $(\eta)$ es una combinación lineal de las variables explicativas

$$
\eta=\beta_{0}+\beta_{1} x_{1}+\ldots \beta_{k} x_{k}
$$

Como en el modelo lineal, las variable regresoras $(X j: j=1,2, \ldots, k)$ están pre especificados y pueden incluir variables cuantitativas, transformaciones de variables, funciones polinomiales, variables "dummy", interacciones de dos o más variables.

3. Una función matemática g(.) monótona e invertible que enlaza la esperanza de la variable respuesta y el predictor lineal y permite linealizar el modelo dado por

$$
g^{-1}\left[E\left(Y / X_{1}=x_{1}, \ldots, X_{k}=x_{k}\right)\right]=\beta_{0}+\beta_{1} x_{1}+\ldots \beta_{k} x_{k} .
$$

En particular, cuando la variable de interés, $Y$, es el resultado de un experimento de Bernoullí, esto es, las observaciones son independientes y sólo pueden tomar los valores $Y=1$ en presencia de la característica de interés, con probabilidad de ocurrencia igual a $\pi$ y $Y=0$ en ausencia de la característica, con probabilidad $1-\pi$. Además, esta variable está explicada por un conjunto de factores (variables explicativas) $X_{1}, X_{2}, \ldots, X_{k}$, las cuales pueden ser categóricas o numéricas; entonces la esperanza, $\pi$, puede estimarse a partir de un modelo de regresión que tiene la forma de una curva sigmoidea dada por la función logística.

Para realizar el ajuste del modelo se utilizará la función de enlace logit, la cual corresponde a la función de enlace canónico de un modelo lineal generalizado para respuesta Bernoullí y se expresa como

$$
\operatorname{Ln}\left[\frac{\pi}{1-\pi}\right]=\beta_{0}+\beta_{1} x_{1}+\ldots \beta_{k} x_{k} .
$$

Una ventaja importante de este modelo, es que a partir de los parámetros estimados se puede calcular un indicador de asociación muy utilizado en el análisis de factores de riesgo relacionados con eventos tales como, por ejemplo, presencia/ausencia de una enfermedad denominado razón de ventajas o más comúnmente conocido como "odds ratio" y denotado como OR, el cual mide la posibilidad o chance de que una enfermedad esté presente si el factor de riesgo está presente. Este indicador es una aproximación al riesgo relativo en estudios transversales en los cuales no es posible estimar directamente los riesgos.

La estimación de parámetros y la evaluación del ajuste del modelo se realiza utilizando la teoría desarrollada para los modelos lineales generalizados.

Previo a la aplicación de los modelos lineales generalizados se realizaron análisis estadísticos bivariados para identificar los posibles factores asociados con la desnutrición crónica y luego se realizaron análisis multivariados utilizando el modelo lineal generalizado para respuesta binaria con función de enlace logit en cual permitirá identificar los factores de riesgo ajustados [5].

\section{Resultados}

La muestra inicial estuvo conformada por 8103 niños menores de 5 años y sus respectivas madres. La mayoría de los niños residían en el campo $(42,2 \%)$ o en ciudades pequeñas $(14,5 \%)$ en el momento de la entrevista, el resto residían en ciudades. El 40,7\% de los hogares de los niños pertenecen a la sierra, $27 \%$ a la selva alta y el resto corresponde a Lima Metropolitana y el resto de la costa. El 42,2\% de estos hogares están ubicados en la zona rural según la clasificación del INEI. La proporción de la muestra correspondiente a cada uno de los 24 departamentos es similar con excepción del Departamento de Lima $(7,8 \%)$ y la Provincia Constitucional del Callao $(0,8 \%)$. 
Aproximadamente las dos terceras partes de las viviendas cuentan con agua potable, 36, $6 \%$ tiene servicios sanitarios dentro de la vivienda y con respecto a la electricidad se observó que las tres cuartas partes de los hogares cuentan con este servicio. Para cocinar, aproximadamente en el $50 \%$ de la viviendas se utiliza el gas GLP para obtener combustible.

El material predominante en las construcciones de las viviendas es la tierra, tanto en paredes $(43,1 \%)$ como en los techos $(51,4 \%)$ y el piso $(45,8 \%)$, esto se explica por que la mayor proporción de la muestra está en el campo y los pueblos pequeños 56,7\%). Solamente una pequeña proporción de las viviendas cuenta con un teléfono $(16,5 \%)$. La cuarta parte de los hogares tiene un refrigerador. Sin embargo, se observa que $70,7 \%$ de estos hogares tiene televisión.

Para medir el nivel de bienestar de los hogares las ENDES calculan el Índice de riqueza. De acuerdo con este índice se tiene que $54,5 \%$ de los niños pertenece a hogares clasificados como pobres o muy pobres, $22,8 \%$ vienen de hogares de clase media y el resto son ricos o muy ricos. En relación a las características de las madres se observó que sus edades oscilan entre 15 y 49 años, con una edad promedio de $29,5 \pm 7,2$ años y su lengua materna es el castellano $(72,1 \%)$, el resto habla quechua, aymara u otra lengua nativa. Aproximadamente, la décima parte de estas mujeres no sabe leer, 34, $2 \%$ tienen grado de instrucción primaria y sólo el 6,4\% tiene instrucción superior. El 66,2\% son esposas del jefe de familia y 7,7\% de ellas son jefes de su hogar. La mayoría de ellas son convivientes $(56,5 \%)$ y sólo $29,3 \%$ están legalmente casadas. El $30,5 \%$ de las madres nunca trabajó, y entre las que actualmente trabajan; 38, $4 \%$ lo hacen en labores agrícolas, $21 \%$ son comerciantes; las demás se dedican a diversas actividades laborales. Con la finalidad de seleccionar los medios de comunicación masiva a los que tiene acceso la mayoría de las madres, de modo que se les pueda informar sobre temas relacionados con salud, higiene, hábitos alimenticios saludables, la ENDES investiga sobre este tema. En particular en la encuesta del 2009, se encontró que más del $50 \%$ escucha radio y ve televisión casi todos los días (56,2\%). Además, se observó que 56,2\% de las madres afirma que lee los periódicos casi todos los días. Pero, en el caso de los diarios sería importante averiguar que diarios leen y cual es el significado que le dan a "leer", puesto que se sabe que la mayoría de las personas en nuestro país no compra diarios y sólo leen la primera página de los diarios en los Puestos de periódicos. Con relación a los niños y niñas menores de 5 años que constituyen las unidades de análisis en este estudio, $51,5 \%$ son de sexo masculino y sus edades varían entre 0 y 59 meses, con una edad promedio de 26, $9 \pm 17,1$ meses.

El análisis de los datos sobre gestación y nacimiento mostró que en el 73,7\% de los niños, la madre tuvo controles pre natales por parte de un personal de salud (médico, enfermera, obstetra, u otro personal entrenado para este tipo de atención. En el 72,1\% de los casos los controles pre natales se iniciaron entre el primer y tercer mes de gestación. La madre fue atendida durante el parto, por un médico en $44,4 \%$ por una enfermera $(50,6 \%)$, o un obstetra $(60,4 \%)$. La décima parte de las mujeres fue atendido por un pariente o amigo u otra persona. El lugar del alumbramiento fue mayoritariamente en un hospital o centro de salud de MINSA (54,6\%), 19, $4 \%$ alumbró en su domicilio y 10,6\% en un hospital de ESSALUD.

La prevalencia de desnutrición crónica es $46,4 \%$ cuando el orden de nacimiento es el 6to o mayor, mientras que si es el primero la prevalencia es la mitad $(23,6 \%)$.Por otro lado, si el niño o niña nació con un peso menor de 2500 grs la prevalencia de desnutrición crónica es de $47 \%$.

En promedio los niños de la muestra lactaron 14,9 $\pm 8,7$ meses. Se observó también que los niños menores de 1 año tuvieron en promedio 6 meses de lactancia, los de un año un promedio de 15 meses y los mayores entre 18 y 20 meses.

Para detectar anemia en los niños y sus madres, en las ENDES se utiliza la medición de hemoglobina. Esta medida constituye un indicador indirecto para deficiencia de hierro. La presencia de anemia constituye un factor de riesgo para el desarrollo sicomotor de los niños y niñas menores de 5 años que se encuentran en proceso de crecimiento. 
En la submuestras estudiada de la ENDES 2009 se encontró que 1855 niños no cuentan con mediciones de hemoglobina $(22,9 \%)$, por lo que la muestra se redujo a 6248 niños. En este grupo se encontró que $42,3 \%$ de los niños presentan algún nivel de anemia (severa, moderada o leve).

El estado nutricional de los niños y niñas menores de 5 años es utilizado internacionalmente como un indicador de desarrollo de los países y constituye uno de los ocho Objetivos del Milenio que el Perú se ha comprometido a cumplir. Esta es la razón por la que el estado nutricional de las madres y sus hijos menores de 5 años es monitoreada en las ENDES, registrándose los pesos, tallas y edades de los niños y niñas menores de 5 años y de las mujeres en edad fértil. Estos datos constituyen los insumos para evaluar el estado nutricional de los niños y niñas.

A partir de las medidas antropométricas se obtuvieron los indicadores: Talla para la edad ( $\mathrm{T} / \mathrm{E})$, peso para la edad $(\mathrm{P} / \mathrm{E})$ y peso para la talla $(\mathrm{P} / \mathrm{T})$. La clasificación del estado nutricional se realizó tomando como base el Patrón de Crecimiento Infantil de la Organización Mundial de la Salud (OMS) difundido internacionalmente el año 2006. Este patrón de referencia se elaboró sobre la base de niñas y niños que estuvieron en un entorno óptimo para el crecimiento: prácticas de alimentación recomendadas para lactantes, niñas y niños pequeños, buena atención de salud, madres no fumadoras y otros factores relacionados con los buenos resultados de salud [9].

Para la expresión e interpretación de los datos antropométricos se calcularon puntuaciones Z (valores tipificados con relación a la población de referencia sugerida por la OMS) para cada uno de los indicadores: peso para la talla $(\mathrm{P} / \mathrm{T})$, talla para la edad $(\mathrm{T} / \mathrm{E})$, peso para la edad $(\mathrm{P} / \mathrm{E})$ e índice de masa corporal (IMC). Luegos se construyó el índice de desnutrición crónica considerando 'puntuaciones por debajo de -2DE del valor $\mathrm{Z}$ de $\mathrm{T} / \mathrm{E}$. Este indicador se considera el más adecuado para evaluar el estado nutricional de los menores de 5 años.[4], [11]

La prevalencia de desnutrición crónica severa estimada fue similar a la obtenida con la muestra total en la ENDES 2009 ((Fig,1)). Notar que, la prevalencia de desnutrición es menor $(4,06 \%)$ en los menores de un año lo cual se explica por el hecho que estos niños y niñas estaban siendo alimentados con la leche materna en el momento de la encuesta. A partir del año de edad la prevalencia es la más alta $(9,52 \%)$, luego tiende a desciende lentamente.

Fig.1. Prevalencia de desnutrición crónica severa (Talla para la edad) en niños y niñas menores de 5 años, según edad actual.

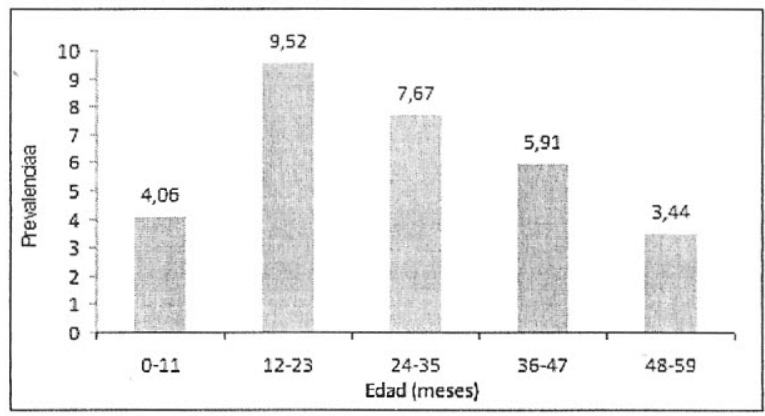

Para el ajuste de los posibles modelos se utilizó un indicador en el cual se incluye desnutrición crónica severa y total por lo que la prevalencia de desnutrición resultó más elevada. El siguiente paso fue realizar un análisis de la relación entre cada uno de los factores y el indicador de desnutrición, observandose que la prevalencia de desnutrición está significativamente asociada con la región $(p<0,001)$, siendo mayor en la sierra $(P: 39,9 \%)$ y en la selva $(P: 25,8 \%)$. Del mismo modo, en la zona rural, la prevalencia de desnutrición crónica $((P: 40,1 \%)$ es significativamente mayor $(p<0,001)$. Las diferencias entre departamentos también son notables Así tenemos que en los departamentos de Huancavelica (53,3\%), Ayacucho (43,7\%), Cajamarca $(42,1 \%)$, Huanuco $(40,6 \%)$ y Pasco $(43,7 \%)$ se observan prevalencias por encima del $40 \%$. Con relación a las características de la madre, se observó la prevalencia de desnutrición crónica está significativamente relacionada con la educación de la madre $(p<0,001)$. Así, se observa que la prevalencia de desnutrición crónica es elevada entre los hijos de mujeres sin 
ningún nivel de instrucción $(55,5 \%)$ y tiende a disminuir a medida que aumenta el grado de instrucción, llegando a casi la tercera parte cuando la madre tiene nivel de instrucción superior $(18,2 \%)$. La prevalencia de desnutrición también está significativamente asociada con la edad de la madre $(p<0,001)$. Entre las más jóvenes y las más adultas la prevalencia tiende a ser mayor $(p<0,001)$. Por otro lado, de acuerdo a la lengua materna (etnia) se observa una mayor prevalencia entre los hijos e hijas de mujeres. Finalmente, se averiguó si la madre trabaja y se observa que la prevalencia de desnutrición crónica es significativamente mayor $(p<0,001)$ entre los hijos e hijas de mujeres que trabajan (31,6\%).

Con respecto a las características de los niños y niñas se observa que la prevalencia de desnutrición crónica es menor entre los niños y niñas menores de un año y se incrementa a partir del primer año de vida. Esto se explicaria por que al dejar la lactancia exclusiva y comenzar a comer otro tipo de alimentos, los niños y niñas están más expuestos a las EDAs lo cual contribuye a la desnutrición de los niños. Esta prevalencia es significativamente mayor entre los niños $(30 \%)$ y entre los que nacieron con bajo peso $(47 \%)$. Por otro lado, se observa también el orden de nacimiento está significativamente asociado con la presencia de desnutrición crónica $(p<0,001)$. Los niños y niñas que son primogénitos presentan la menor prevalencia de desnutrición $(23 \%)$ y esta se va incrementando a medida que el orden de nacimiento se incrementa.

Con relación a la presencia de anemia, se observa que cuanto más severa es la anemia entre los niños y niñas, la prevalencia de desnutrición crónica se incrementa significativamente $(p<0,001)$.

Una vez identificados los factores relacionados con la prevalencia de desnutrición crónica, se realizó un análisis multivariado mediante el ajuste de un modelo lineal generalizado (MLG) logit para respuesta binaria. En la tabla 1, se muestran los factores medio ambientales, de la madre y de los niños y niñas menores de 5 años asociados significativamente con la prevalencia de desnutrición crónica $(p<0,001)$.

Entre las características relacionadas con el entorno se encontró que la posibilidad de presentar desnutrición crónica es significativamente mayor entre los niños y niñas que residen en la zona rural(OR:1,30;IC:1,1-1,5. Por otro lado, se tiene que la chance de presentar desnutrición crónica aumenta significativamente a medida que se incrementa el índice de pobreza, alcanzando su máximo en el quintil de pobreza extrema (OR:5,8;IC:3,6-9,1). 
Tabla1. Factores asociados con la desnutrición crónica en niños y niñas menores de 5 años. OR ajustados e Intervalos de confianza.

\begin{tabular}{|c|c|c|c|c|}
\hline \multirow{2}{*}{ FACTORES ASOCIADOS } & \multirow{2}{*}{$\begin{array}{l}\text { COEFICIENTE } \\
\text { DE REGRESIÓN }\end{array}$} & \multirow{2}{*}{ ORAjustado } & \multicolumn{2}{|c|}{ 1.C. $95,0 \%$ para ORajustado } \\
\hline & & & L. Inferior & L. superior \\
\hline \multicolumn{5}{|c|}{ CARACTERISTICAS DE LA MADRE } \\
\hline \multicolumn{5}{|c|}{ Grado do instruccion } \\
\hline Superior & & 1,00 & & \\
\hline Secundaria & 0,119 & 1,13 & 0,89 & 1,42 \\
\hline Primaria & 0,429 & 1,54 & 1,19 & 1.98 \\
\hline Sin educación & 0,833 & 2,30 & 1,60 & 3,31 \\
\hline \multicolumn{5}{|l|}{ Edad } \\
\hline $35-49$ & & 1,00 & & \\
\hline $20-34$ & 0,219 & 1,24 & 1,05 & 1,48 \\
\hline $15-19$ & 0,639 & 1,90 & 1,36 & 2,63 \\
\hline \multicolumn{5}{|l|}{ Lengua Matema } \\
\hline Castellano & & 1,00 & & \\
\hline Quechua/aymara & 0,325 & 1,38 & 1,20 & 1,59 \\
\hline Otras lenguas nativas & 0,777 & 2,18 & 1,56 & 3,03 \\
\hline \multicolumn{5}{|c|}{ CARACTERISTICAS DEL NIÑO } \\
\hline \multicolumn{5}{|l|}{ Sexo } \\
\hline Femenino & & 1,00 & & \\
\hline Masculino & 0,145 & 1,16 & 1,02 & 1,31 \\
\hline \multicolumn{5}{|l|}{ Peso al nacer } \\
\hline Normal & & 1,00 & & \\
\hline Вајо & 0,815 & 2,26 & 1,80 & 2,83 \\
\hline \multicolumn{5}{|l|}{ Orden de nacimiento } \\
\hline $1^{\circ}$ & & 1,00 & & \\
\hline $2^{\circ}-3^{\circ}$ & 0,272 & 1,31 & 1,11 & 1.56 \\
\hline $4^{\circ}-5^{\circ}$ & 0,574 & 1,78 & 1,42 & 2,21 \\
\hline $6^{\circ}$ a más & 0,661 & 1,94 & 1,47 & 2,55 \\
\hline \multicolumn{5}{|l|}{ Edad actual (años) } \\
\hline Menos de 1 & & 1,00 & & \\
\hline De 1 a menos de 2 & 0,912 & 2,49 & 1,99 & 3,11 \\
\hline De 2 a menos de 3 & 0,909 & 2,48 & 1,96 & 3,15 \\
\hline De 3 a menos de 4 & 0,954 & 2,60 & 2,03 & 3.33 \\
\hline De 4 a menos de 5 & 0,635 & 1,89 & 1,46 & 2,43 \\
\hline \multicolumn{5}{|l|}{ Nivel de anemia } \\
\hline Sin anemia & & 1,00 & & \\
\hline Leve & 0,220 & 1,25 & 1.07 & 1.45 \\
\hline Moderada & 0,162 & 1,18 & 0,98 & 1,41 \\
\hline Severa & 0,823 & 2,28 & 1,15 & 4,50 \\
\hline \multicolumn{5}{|c|}{ CARACTERISTICAS MEDIO AMBIENTALES } \\
\hline \multicolumn{5}{|c|}{ Zona de residencia } \\
\hline Urbana & & 1,00 & & \\
\hline Rural & 0,262 & 1.30 & 1,11 & 1.53 \\
\hline \multicolumn{5}{|l|}{ Indice de riqueza } \\
\hline Muy rico & & 1,00 & & \\
\hline Rico & 0,612 & 1,84 & 1,17 & 2.91 \\
\hline Medio & 0,887 & 2,43 & 1,57 & 3,75 \\
\hline Pobre & 1,327 & 3,77 & 2,42 & 5,86 \\
\hline Muy pobre & 1,749 & 5,75 & 3,63 & 9,11 \\
\hline
\end{tabular}

En relación a las características de la madre (Tabla 1) se observa que a medida que disminuye el grado de instrucción de la madre se incrementa la chance de que el niño o niña presente desnutrición crónica, alcanzando su máximo cuando esta no tiene ningún nivel de instrucción (OR:2,3;IC:1,6-3,3). Tomando como referencia a las madres, a mayor edad se observa que la posibilidad de presentar desnutrición crónica se incrementa a medida que las madres son más jóvenes y es máxima entre las mujeres entre 15 y 19 años de edad $(O R: 1,9 ; I C: 1,4-2,6)$. Lo mismo ocurre con la lengua materna, la chance se hace mayor cuando la madre habla lenguas nativas (no castellano), (OR:2,2;IC:1,6-3,0).

Finalmente, el análisis de las características de los niños y niñas muestra que el bajo peso al nacer $(O R: 2,3 ; I C: 1,8-2,8)$ y el sexo masculino (OR;1,2; IC:1,1-1,3) aumentan significativamente la chance de presentar desnutrición crónica.

Si el niño presenta anemia la posibilidad de presentar desnutrición crónica se incrementa a medida que la anemia es más aguda, alcanzando su máximo en presencia de anemia severa (OR:2,28; IC:1,15-4,50). Lo mismo ocurre con el orden de nacimiento, la chance se incrementa a medida que el niño o niña tiene un orden de nacimiento mayor (ver Tabla 1) .

De acuerdo a la edad del niño o niña también vemos que a medida que va aumentando la edad del niño o niña se incrementa la posibilidad de presentar desnutrición crónica.

\section{Conclusiones}

Como hemos podido observar a partir de la revisión bibliográfica, la nutrición en los primeros cinco años de vida juega un papel muy importante en el crecimiento físico y el desarrollo 
intelectual del ser humano. Una adecuada nutrición va a favorecer tanto el crecimiento corporal del niño; como el desarrollo de sus capacidades cognoscitivas y lo harán más resistente a las enfermedades.

Una de las preocupaciones del Ministerio de Salud, es que a partir del 2002 a nivel nacional, la tasa de desnutrición no ha sufrido cambios, a pesar de los esfuerzos realizados para mejorar el estado nutricional de la población.

La investigación permitió confirmar que existen factores ambientales, los cuales influyen significativamente en el estado nutricional de los niños y niñas menores de 5 años en el Perú. Así pudimos comprobar que la prevalencia de desnutrición crónica es significativanıente mayor en la zona rural y en niños pertenecientes a hogares pobres y muy pobres, esto concuerda con estudios realizados por CEPAL entre 2003 y 2006 a nivel de America Latina y el Caribe del año 2006. [5]

En relación a los factores asociados con la madre, también se observa que la edad de la madre es un factor importante, pues entre los niños que tienen madres muy jóvenes o muy adultas la prevalencia de desnutrición crónica es significativamente mayor.

Entre las características de los propios niños y niñas, se encontró que en sexo masculino la prevalencia es significativamente mayor. Del mismo modo, el presentar bajo peso al nacer aumenta su chance de presentar desnutrición crónica y el riesgo aumenta significativamente con el orden de nacimiento.

En relación a la lactancia materna, se ha observado que el tiempo promedio de lactancia es de aproximadamente 15.meses con una variación promedio de 8 meses. Entre los niños y niñas que en el momento de la encuesta tenían menos de dos años, se encontró que la lactancia era en promedio de 6 meses.

Otro aspecto que se observó es que la prevalencia de desnutrición en los niños y niñas es es máxima entre uno y dos años de edad en que los infantes comienzan a caminar y ya consumen alimentos sólidos, lo cual puede exponerlos más a contraer enfermedades como las EDAs y las IRAs por lo que pierden peso y desarrollan adecuadamente.

Finalmente, la mayoria de los factores de riesgo identificados pueden ser controlados con la diminución de la pobreza y la mejora en la educación de la población, un adecuado control pre natal y una mejora de la calidad de vida de las madres, especiamente en la zona rural. 


\section{REFERENCIAS BIBLIOGRÁFICAS}

[1] Barreto Penié Jesús. Mala Nutrición Por Defecto: Historia, Estado Actual y Perspectivas. Rev Cubana Med 1999; 38(1):3-6.

[2] INEI (2007). Análisis de los factores subyacentes asociados a la desnutrición crónica infantil en el Perú. Centro de Investigación y Desarrollo (CIDE).

[3] INEI 2009). Encuesta Demográfica y de Salud Familiar-ENDES Continua. Informe Principal.

[4] Kaplan S.(1991). Growth. Rudolphs Pediatrics. Abraham Rudolph, 19th Edition, Prentice Hall Internacional lnc. 129-139.

[5] McCullagh, P.; Nelder, J. A. (1989). Generalized Linear Models. Chapman and Hall, London, second edition.

[6] Nelder, J. A.; Wedderburn, R. W. M. (1972). Generalized linear models. Journal of the Royal Statistical Society, A, 135, 370-384.

[7] OMS, Serie de Informes Técnicos. El estado físico: uso e interpretación de la antropometría. Ginebra, 1995.

[8] Organización Panamericana de la Salud /Organización Mundial de la Salud. Desnutrición Infantil en las Américas: Cumplimiento de los Objetivos de Desarrollo del Milenio. Washington DC:WHO; 2008.

[9] Martínez Rodrigo y Fernández Andrés (2006). Desnutrición infantil en América Latina y el Caribe. Boletín de la infancia y adolescencia sobre el avance de los objetivos de desarrollo del Milenio. División de de Desarrollo Social de la CEPAL. Desafíos Número 2

[10] UNICEF (United Nations Children's Fund) (1988). The State of World Children 1998: Focus on Nutrition. Oxford University Press.

[11] World Health Organization. Working Group (1986). Use and interpretation of anthropometric indicators of nutritional status. Bull of WHO 64 (6): 929-41. 